\title{
Polarimetric Current Sensor USING A IN-LINE 22.5 DEGREE FARADAY ROTATOR
}

\author{
F. Briffod, L. Thévenaz, P.-A. Nicati, A. Kueng, Ph. Robert \\ EPFL Swiss Federal Institute of Technology, Laboratory of Metrology, CH-1015 Lausanne, Switzerland \\ Tel : (++41 21) 6937606 Fax : (++41 21) 6932614 E-mail : fabien.briffod@epfl.ch
}

Abstract : This paper present a polarimetric fiber optics current sensor using a 22.5 degree Faraday rotator and a Faraday rotation mirror. The objective of this sensor is to be low-cost, high accuracy and easy to manufacture.

\section{INTRODUCTION}

Fiber optic current sensors have some advantages compared to the classical current transformer. The intrinsic insulation of the optical fiber is an important improvement for high voltage installations. They show a high bandwidth that makes the observations of harmonics and transients possible and offer a total immunity to stray magnetic fields.

Producing commercial optical current sensors is still challenging and prototypes are developed in three different flavors: bulk, fiber interferometric and fiber polarimetric sensors. Bulk current sensors [4] are made using high Verdet's constant crystals, resulting in an excellent sensitivity, but are subject to alignment and temperature drifts. Optical fibers show a lower Verdet's constant, but increasing the number of turns of fiber wound around the conductor results in an improved sensitivity. The interferometric configuration using a Sagnac interferometer [1] measures the non-reciprocal phase shift with a high accuracy, but the main reported problem is the high vibration sensitivity. The polarimetric method measures the rotation of a linear polarization $[2,3]$. Such sensors require a precise orientation of the analyzer. And as a general feature all fiber sensors are sensitive to the variations of the sensing fiber birefringence.

A novel sensor configuration is reported that solves all these problems to a wide extent. In addition, it has the key advantage to require no adjustment on optical elements of any kind and its accuracy is poorly dependent on the optical elements tolerance. This was possible through the introduction of a in-line 22.5 degree Faraday rotator (or $22.5+\mathrm{n} \times 90$ degree rotator), as shown in figure 1 .



Fig. 1 : Schematic diagram of the polarimetric current sensor using an in-line 22.5 degree Faraday rotator

\section{DESCRIPTION}

The optical configuration is simple and based on the back-and-forth propagation through all successive optical elements, the position of reciprocal and non-reciprocal elements being crucial. First let assume that the electrical current is zero. The light is linearly polarized by the polarizer and experiences a 22.5 degree rotation through the Faraday 
rotator. The sensing fiber is mechanically twisted and is thus predominantly circularly birefringent [6], so that the light remains linearly polarized while propagating through the sensing fiber. The light is reflected back by the mirror and keeps its linear polarization. Just before the Faraday rotator on the way back, the polarization has the same orientation as in the forward propagation (configuration A). After the additional 22.5 degree rotation, the polarization is oriented at 45 degree with respect to the polarizer axis.

The linear polarization is therefore just set at the half-transmitting point of the polarizer, so that any small rotation of the polarization - like that caused by an electrical current - results in a linear variation of the transmitted intensity.

When the electrical current $I_{e l}$ is non-zero, the Faraday rotation $\varphi(t)$ due to the electrical current is after a back-andforth propagation through the sensing fiber:

$$
\varphi(t)=2 N V I_{e l}(t)
$$

where $\mathrm{V}$ is the Verdet's constant. In the case of a fiber coil, this angle is also multiplied by the number $\mathrm{N}$ of turns of fiber around the conductor.

The total rotation experienced by the linearly polarized light is $\varphi_{t o t}=\pi / 4+\varphi(t)$ and the output intensity is given by:

$$
I(t)=I_{0} \cos ^{2}\left(\varphi_{t o t}\right)=\frac{I_{0}}{2}+\frac{I_{0}}{2} \cos \left(2 \varphi_{t o t}\right)=\frac{I_{0}}{2}-\frac{I_{0}}{2} \sin \left(4 N V I_{e l}(t)\right)
$$

The sensor is thus set to the optimal operating point for sensitivity and linearity. This sensor being designed for AC current measurement, it is possible to filter the DC and AC components:

$$
I_{D C}=I_{0} / 2 \quad \text { and } \quad I_{A C}=-\frac{I_{0}}{2} \sin \left(4 N V I_{e l}(t)\right)
$$

so that a normalization of the AC value by the DC value makes it independent of fluctuations of the light intensity and of the optical losses. Finally, we simply obtain:

$$
I_{e l}(t)=-\frac{1}{4 N V} \arcsin \left(\frac{I_{A C}(t)}{I_{D C}}\right) \approx-\frac{1}{4 N V} \frac{I_{A C}(t)}{I_{D C}} \quad \text { for } I_{A C}(t) \ll I_{D C}
$$

If the Faraday rotator is not exactly at 22.5 degree the operating point is slightly shifted off the center of the linear response. The response becomes therefore partially nonlinear and can be efficiently approximated using a $2^{\text {nd }}$ or $3^{\text {rd }}$ order polynomial, so that using a non-ideal rotator causes no real penalty. This correction has also to be performed for large currents even when using an ideal Faraday rotator. In all cases the sensor response remains independent from variation of intensity and losses and a calibration of the sensor is only needed to correct the possible nonlinearity.

But a key advantage of this configuration remains that no orientation of the polarizer and the Faraday rotator is required, making this sensor easy to manufacture and intrinsically stable.

To make the system even more immune to birefringence, it is possible to substitute the standard mirror by a Faraday rotation mirror [7] (configuration B) at the expense of a less cost-effective setup. The sensing fiber is never strictly non-birefringent, so that the use of a Faraday mirror cancels the effect of birefringence and its variations. In absence of electrical current the polarization of the backward propagating light is, at any point, orthogonal to the polarization of the light traveling in the forward direction. For instance if a lightwave travels along the fast axis of a birefringent fiber in the forward direction, it travels along the slow axis in the backward direction, making the effect of any birefringence canceled.

The operating point of such a sensor is no longer at 45 degree but shifts to 135 degree. This only changes the sign of the scaling factor. 
If the Faraday mirror does not show the ideal total rotation of 90 degree, the compensation of the birefringence is no longer perfect and small fluctuations are observed. The operating point is in addition biased but, as mentioned above, this does not reduce the sensor accuracy if a calibration is performed.

When an electrical current is applied, the additional non-reciprocal rotation remains small, so that the birefringence remains compensated to a wide extent, at least to first order.

\section{RESULTS}

The setup uses a $1311 \mathrm{~nm}$ multimode laser. At this wavelength the rotation angle of the in-line Faraday rotator is equal to 22 degree. The coupler splitting ratio is 50/50 and the polarizer has an extinction ratio of $30 \mathrm{~dB}$. The sensing fiber is made from 45 turns of mechanically-twisted fiber and a Faraday rotation mirror with a $1302 \mathrm{~nm}$ nominal wavelength is used. The bandwidth of the sensor has been set at $600 \mathrm{~Hz}$ in order to observe overtones and transients.

The figure 2 represents a measurement showing the raw optical-electrical transfer function. The response is nonlinear for high current, as expected by the theory. The noise level is approximately $700 \mathrm{~mA}$. Our current source could not deliver more than $2 \mathrm{kA}$, but current up to $4 \mathrm{kA}$ could be also measured. Using a $3^{\text {rd }}$ order polynomial as transfer function was observed to perfectly compensate the nonlinearity. In addition the configuration B using a Faraday rotation mirror is measured to be greatly less sensitive to temperature variation than the configuration $\mathrm{A}$ using a standard mirror, as expected.

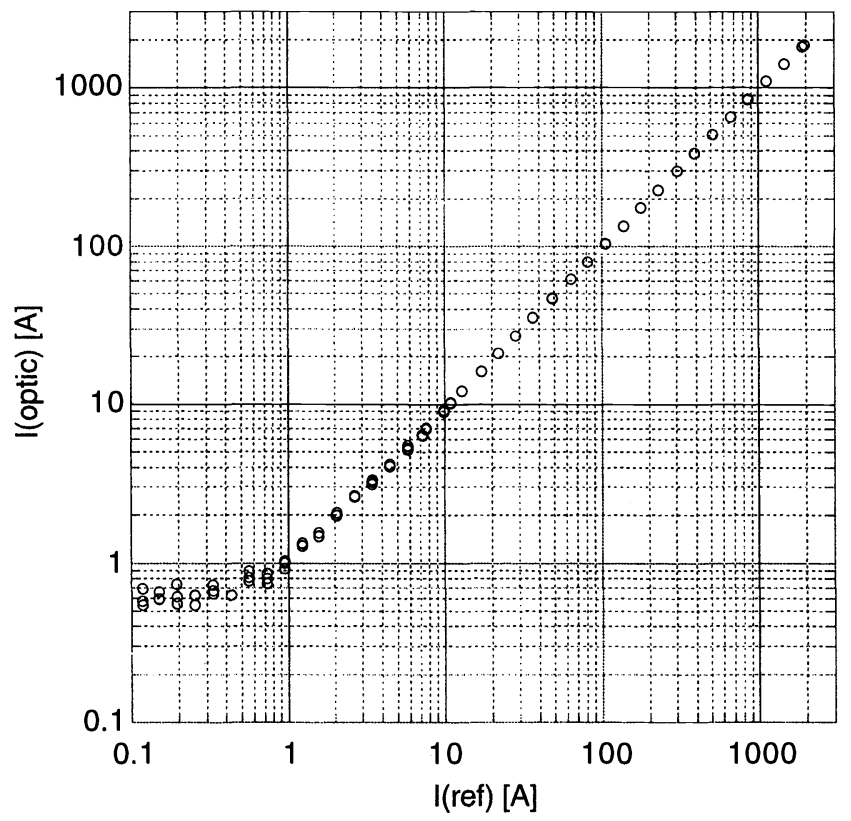

Fig. 2 : Sensor optical-electrical transfer function, showing the noise floor level and the possibility to measure over more than 3 decades.

\section{DISCUSSION AND CONCLUSION}

This sensor offers some important improvements: first it is a self-stable configuration and no optical adjustment is needed, resulting in accuracy independent on manufacturing issues. Then, the use of a non-ideal Faraday rotator and mirror does not significantly reduce the accuracy. Finally, this configuration compensates the residual linear birefringence in the sensing fiber, the temperature variation of the fiber circular birefringence and is greatly immune to vibrations. 


\section{BiBLIOGRAPHY}

[1] P.-A. Nicati, Ph. Robert, "Stabilized current sensor using Sagnac interferometer", Journal of Physics, Vol. 21, No. 8; pp. 791-796, August 1988

[2] J. W. Dawson, T. W. MacDougall, E. Hernandez, "Verdet Constant Limited Temperature Response of FiberOptic Current Sensor”, IEEE Photonics Technology Letters, Vol. 7, No. 12, pp. 1468-1470, December 1995

[3] R. L. Patterson, A. H. Rose, D. Tang, G. W. Day, "A fiber-optic current sensor for aerospace applications", IEEE Aerospace and Electronic Systems Magazine, Vol. 5, No. 12, pp. 10-14, December 1990

[4] A. H. Rose, M. N. Deeter, G. W. Day, "Submicroampere-per-root-hertz current sensor based on the Faraday effect in Ga:YIG”, Optics Letters, Vol. 18, No. 17, pp. 1471-1473, September 1, 1993

[5] D. Tang, A. H. Rose, G. W. Day, S. M. Etzel, "Annealing of Linear Birefringence in Single-Mode Fiber Coils: Application to Optical Fiber Current Sensor", Journal of lightwave technology, Vol. 9, No. 8, pp. 1031-1037, August 1991

[6] R. Urlich, A. Simon, "Polarization optics of twisted single-mode fibers", Applied Optics, Vol. 18, No. 13, pp. 2241-2251, July 1, 1979

[7] N. C. Pistoni, M. Martinelli, "Vibration-insensitive fiber-optic current sensor", Optics Letters, Vol. 18, No. 4, pp. 314-316, February 15, 1993 